\title{
Ultrasound-guided radiofrequency for chronic shoulder pain: preliminary report
}

Rita Torgal, Joana Pinto, Maria do Céu Loureiro, José Pedro Assunção

Chronic Pain Unit, Anaesthesiology Department, Centro Hospitalar Tondela-Viseu, Portugal

\section{Background and Goal of Study:}

Chronic shoulder pain is a frequent cause of suffering and impaired quality of life. Treatment includes multimodal analgesia, physical therapy and interventional procedures such as suprascapular nerve block and pulsed radiofrequency.

The objective of this prospective study is to evaluate efficacy of ultrasound-guided pulsed radiofrequency of suprascapular nerve for chronic shoulder pain in a clinical setting.

\section{Materials and Methods:}

- After ethical approval, the following inclusion criteria were used:

$\checkmark$ previous pain intensity reduction to ultrasound-guided suprascapular nerve block with local anaesthetic and steroids superior to $50 \%$.

- Patients were submitted to ultrasound-guided (Siemens Acuson P 300 , linear probe $12 \mathrm{MHz}$ ) radiofrequency of suprascapular nerve (22 G 60cm needle, 2 cycles, during 180 seconds, temperature < $\left.42^{\circ}\right)$.

- The primary outcome were efficacy using numerical pain rating scale registered before, 1 and 3 months after. Patient motor function (abduction, flection and extension) measured using a goniometer.

- The secondary outcome was patient global satisfaction.

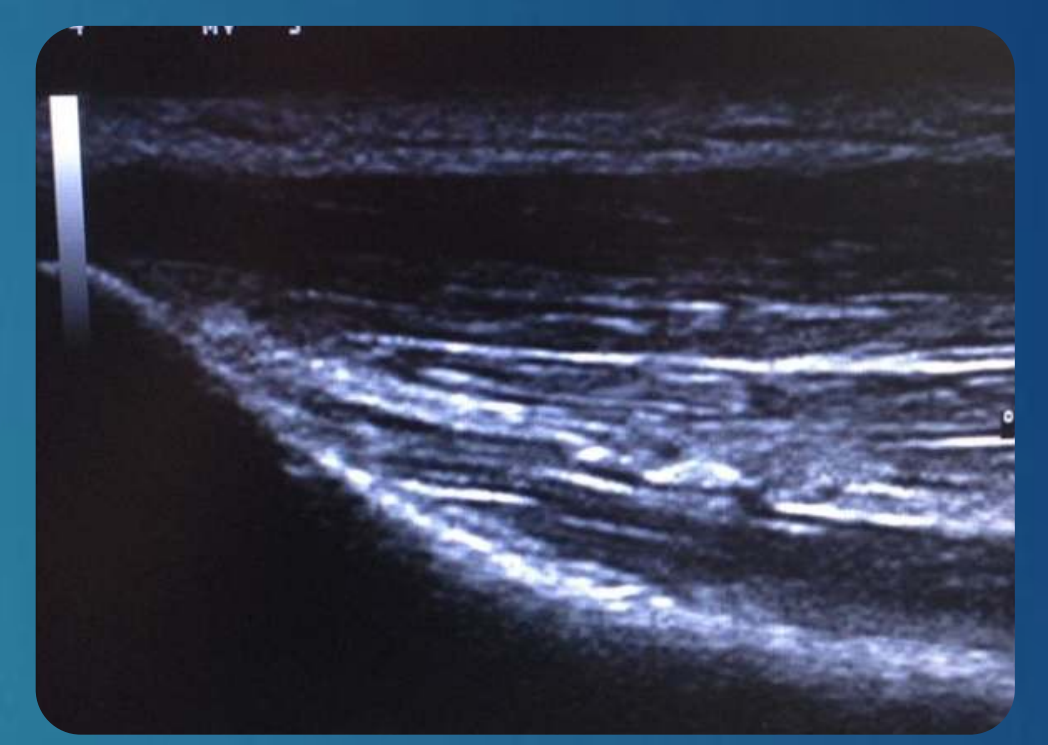

Figure 1: Sonoanatomy

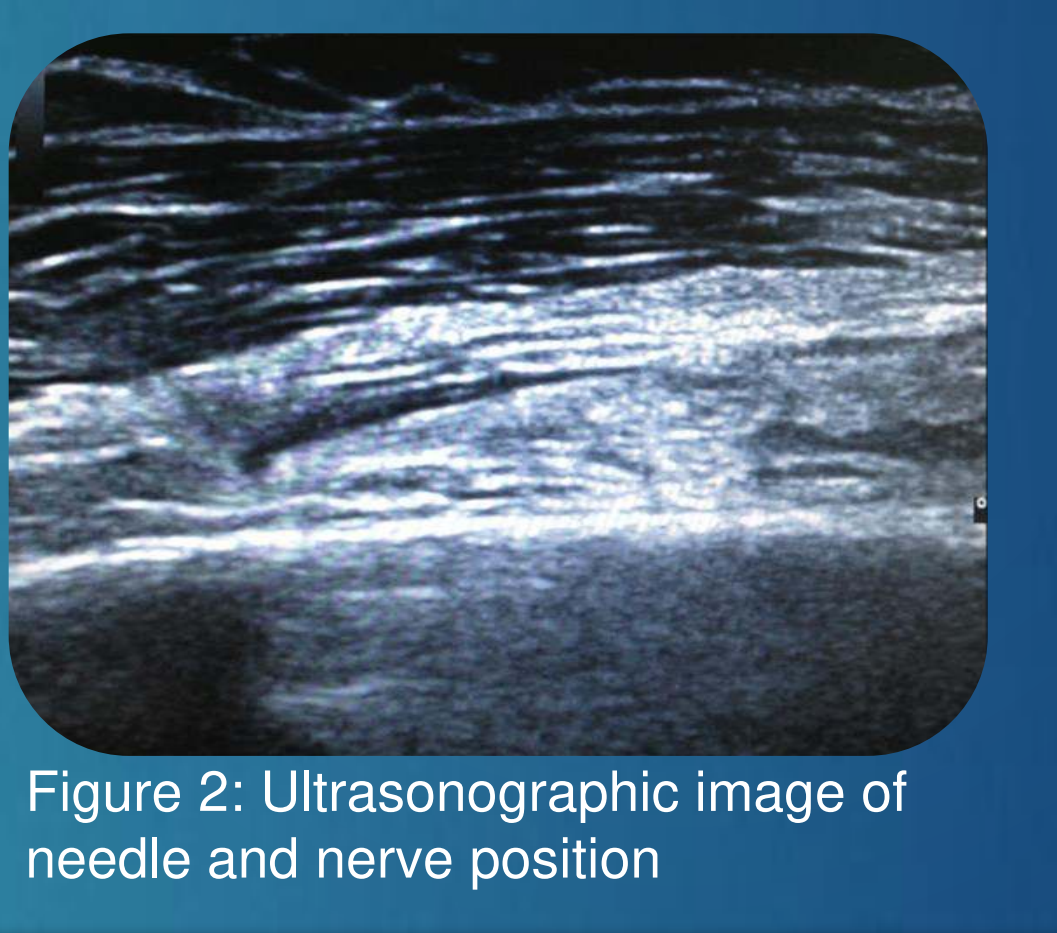

Results and Discussion:

- A total of 8 patients were included, $87.5 \%$ were female, with a mean age of 59.9 years.

- Patients presented significant pain reduction (NPRS score was reduced by at least $30 \%$ ), functional improvement and high levels of satisfaction. No adverse effects were recorded. Patients presented significant pain reduction (NPRS score was reduced by at least 30\%) functional improvement and high levels of satisfaction.
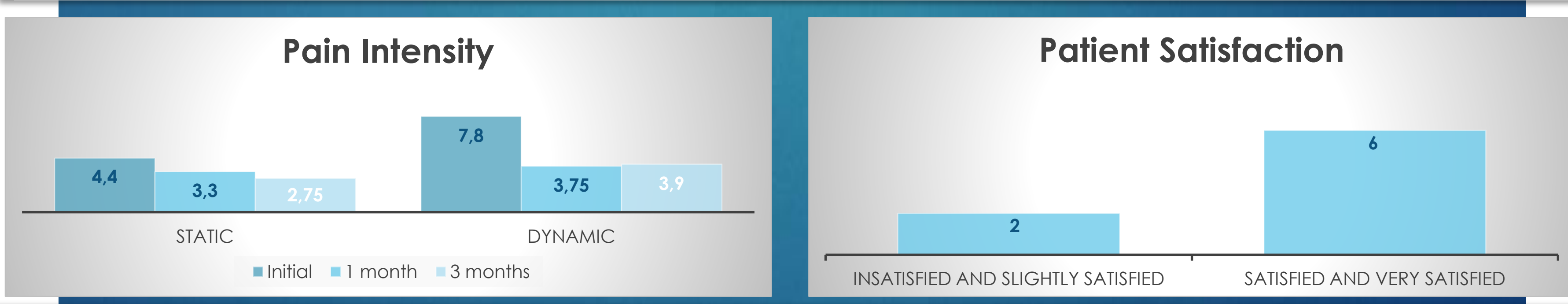

\section{Conclusions:}

Our study concludes that ultrasound-guided pulsed radiofrequency of suprascapular nerve is safe and effective with pain reduction for at least 3 months. Therefore this technique represent a valid analgesic alternative to the treatment of chronic shoulder pain. Future research is warranted for validating these results.

References: Chua NH, Vissers KC, Sluijter ME. Pulsed radiofrequency treatment in interventional pain management: mechanisms and potential indications-a review. Acta Neurochir (Wien). 2011;153(4):763-71. 